\title{
Genetic based optimisation of the design parameters for an array-on-device orbital motion wave energy converter
}

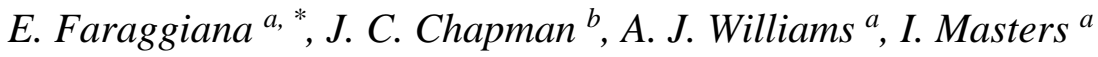 \\ a Energy and Environment Research Group, Zienkiewicz Centre for Computational Engineering, \\ College of Engineering, Swansea University, emilio.faraggiana@alice.it, \\ alison.j.williams@swansea.ac.uk, i.masters@swansea.ac.uk \\ ${ }^{b}$ Marine Power Systems Ltd., Swansea, UK, contact@marinepowersystems.co.uk
}

Abstract—:

Optimisation of Wave Energy Converters (WECs) is a very important topic to obtain competitive devices in the energy market. Wave energy is a renewable resource that could contribute significantly to a future sustainable world. Research is on-going to reduce costs and increase the amount of energy captured. This work aims to optimise a WaveSub device made up of multiple floats in a line by investigating the influence of 6 different design parameters such as the number of floats. Here we show that a multi-float configuration of 6 floats is more competitive in terms of Levelised Cost Of Energy (LCOE) compared to a single float configuration with a LCOE reduction of around $21 \%$. We demonstrate that multi-float configurations of this device reduce the LCOE especially because of the reduction of grid connection, installation, control and mooring costs. From the power capture perspective, optimised multi-float configurations still have similar capacity factors to the single float configuration. This research gives important indications for further development of the WECs from an optimisation perspective. These promising results show that more complex, optimized, multi-float configurations could be investigated in future. Keywords-Renewable energy; wave energy; optimisation; LCOE

\section{Introduction}

Research in wave energy has been growing during this century due to the increased need of renewable resources (Neill and Hashemi, 2018). Different types of Wave Energy Converters (WECs) have been developed based on their working principle and on the location (Falcão, 2014; Aqua-RET, 2019). The Marine Power Systems Ltd. (MPS) WaveSub device (Chapman et al., 2017) under consideration in this paper is an off-shore point absorber characterized by a reactor and a floater which are completely submerged. Its working principle can be found in (Faraggiana et al., 2019) while a multi-float WaveSub is described in (Faraggiana et al., 2020). Float orbital motion is converted into electricity thanks to the PTO lines that are connected to an hydraulic PTO on 
steady base. The multifloat device can be considered to be an array-on-device and several multifloat devices can be organised into an array, saving costs through economies of scale. The optimisation of WEC arrays will play an important role for the development of the wave energy sector. The cost of energy of this technology is currently too high compared to other ways of grid connected energy production and there is the need to find a credible cost reduction pathway.

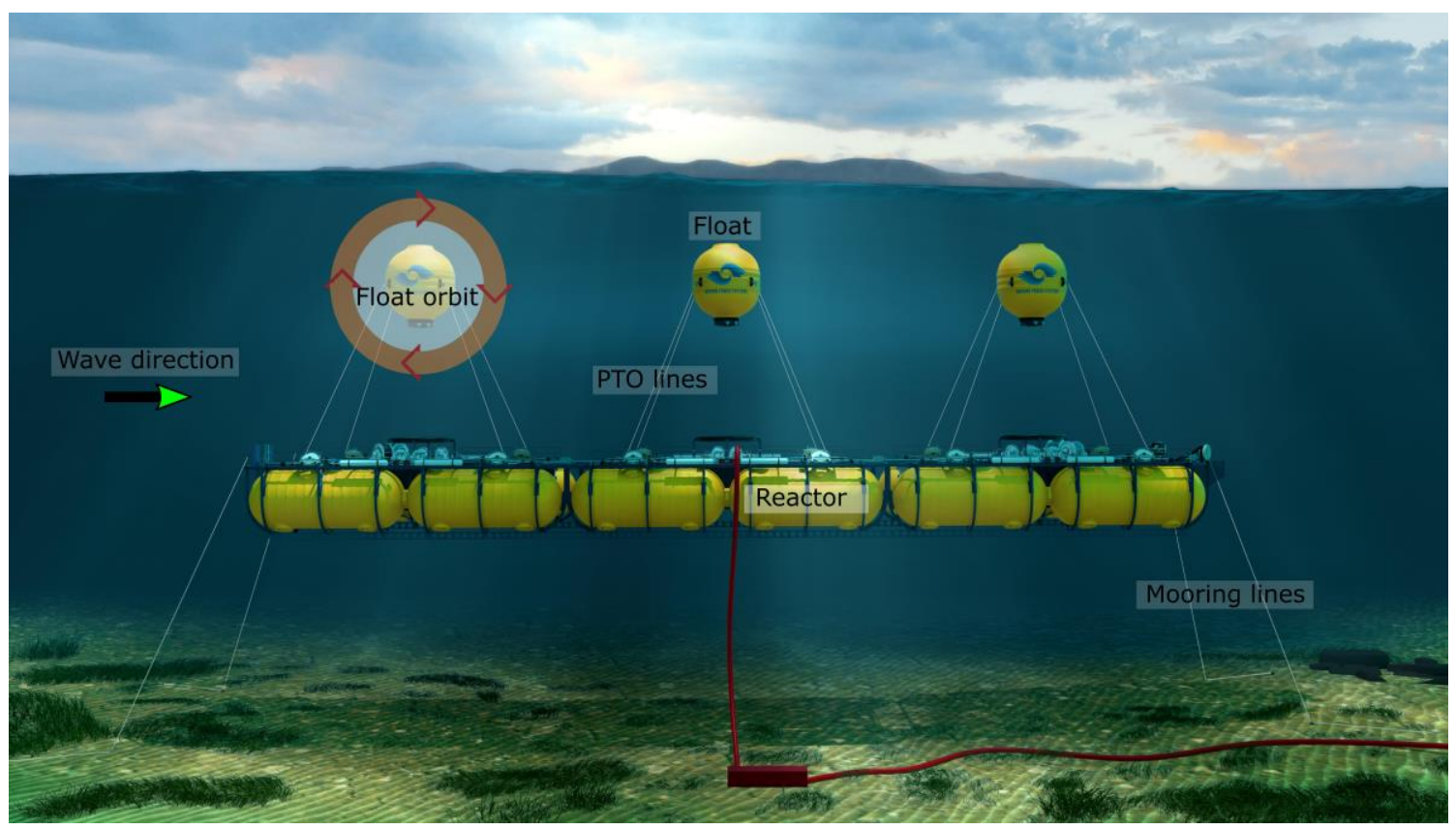

Figure 1: A digital 3 multi-float WaveSub device during operating conditions. (Single column image)

A numerical model was used to assess the energy capture performance of the wave device. In this section we review previous work on array performance modelling, many of which use the Boundary Element Method (BEM) to enable calculation of the hydrodynamic forces acting on a WEC with arbitrary shape, configuration and layout. The focus of this review is work that has attempted to optimise array design either by parametric study or optimisation algorithms. A small array of four point absorbers connected to a moored disk has been optimized as a function of incident wave direction, separating distance between the absorbers and the PTO damping using a BEM approach (Zhang et al., 2016). All these factors have a great influence in the power absorption and have influence between each other. Looking in more detail at the power absorption as a function of the PTO damping, the power absorption first increases and then it starts to decrease slowly. It shows that there is an optimal PTO damping coefficient for each wave frequency. Different array layout were analysed in the work of Zhang. In particular, they found that when a square configuration was compared with rhombus and triangular configurations it gave better results in terms of power production and less sensitivity to the incident wave direction.

A BEM approach has been used also in the work of (Borgarino, Babarit and Ferrant, 2012) in which a parametric analysis of a wave farm has been carried out, observing the influence of some parameters on the power production. In particular, the influence 
of the device separation on the mean absorbed power is reduced if the PTO damping is optimized and if the WEC has a large power capture bandwidth. In this research a WEC farm between 9 and 25 devices has been considered thanks to the use of an accelerated hydrodynamic computation option available within Aquaplus (Delhommeau, 1993). However, generally the computational time required to simulate large WEC arrays is impractical using a BEM approach. When large WEC arrays are considered, simplifying assumptions are necessary such as small and vertical axisymmetric bodies oscillating in one single or double modes of motion (Mavrakos and Koumoutsakos, 1987; Mavrakos, 1991; Mavrakos and McIver, 1997). Most of the research currently available is related to point absorbers and Oscillating Wave Surge Converters (Child, 2011; Sarkar, Renzi and Dias, 2014; Zhang et al., 2016). Oscillating Wave Surge Converters are a shallow water flap type device that operate as a terminator for the waves and consequently devices positioned towards the front of the WEC farm generally have a better performance than the ones at the back (Sarkar, Renzi and Dias, 2014). Further work showed that a WEC farm staggered configuration in a 'bowl' or 'chevron' configuration is shown to be more efficient than a linear configuration (Noad and Porter, 2015). In (McGuinness and Thomas, 2016) the optimisation of a linear array configuration was related to the angle of the direction of the wave train and the position of the wave devices. The spacing between the WECs in a linear array has been also analysed and it has been found that the best configuration is not when the devices are equally spaced. In-fact an asymmetric solution can optimize the energy production. An optimized solution shows the devices closer together in the front of the array due to the positive effect of the radiated field. It is demonstrated that a single row of point absorbers perpendicular to the incident wave can produce $50 \%$ of the incident power if the bodies are operated in one mode (heave) while it could produce all the energy that comes from the waves if operated in two modes (Budal, 1977). Moreover, when the distance between the WECs is the wavelength and the number of devices tends to infinity the power absorption may increase to a maximum factor of $\pi$ when compared to a configuration with isolated WECs. This work is limited because it used harmonic analysis and in practice ocean waves will produce a significant averaging effect and a slow fluctuation of the interaction factor.

In the PhD thesis of Child (Child, 2011) an optimisation of an array configuration of point absorbers is considered using the direct matrix method. Two optimisation methods have been used to find the best array configurations: a heuristic approach called the Parabolic Intersection (PI) method and the Genetic Algorithm (GA). In particular, each method was used to maximize the power produced with a given wave regular frequency and direction. This method enables only simplified calculation but with the advantage of being very fast. It is based on the fact that the constructive and destructive interference is given where the interactive wave field (scattered and radiated) are in or out of phase with the incident wave field (Child and Venugopal, 2009). In particular, the strongest interference will be the closest in phase position to the device. The in-phase curves are approximated with parabolas. These parabolas shapes are constant because the relative phase keeps constant over time. 
71 A farm of several hundreds of point absorbers can be optimized very efficiently using a multi-scattering method as described in 72 (Göteman et al., 2015). This method has been then extended to allow for WECs with different dimensions (Göteman, 2017). A 73 hybrid array is optimized in (Giassi et al., 2017).

74 A coupling between BEM with other methods is also possible. For example semi-analytical array models can be coupled with BEM to save computational time (Chakrabarti, 2005; McNatt, Venugopal and Forehand, 2013).

Chakrabarti proposed an hybrid method that combines the BEM and the multiple-scattering method (Chakrabarti, 2005). This hybrid method overcomes the limitation of the geometry of the multiple-scattering method and at the same time it has less computational time than the BEM.

McNatt proposed in a similar way to Chakrabarti the coupling between the direct matrix method and the BEM (McNatt, Venugopal and Forehand, 2013). McNatt developed a MATLAB open-source tool named mwave (McNatt, 2019). (Sharp et al., 2017) applied mwave to an array optimisation of fixed oscillating water columns.

Finally a coupled numerical model combining a wave propagation model (MILDwave) and a BEM (WAMIT) has been used by Stratigaky (Stratigaki, 2014). A large number of WEC point absorbers (25) has been considered in the optimisation comparing experimental and numerical results.

\subsection{Aims of this work}

This paper aims to optimise a WEC array of a linear multi-float configuration of the WaveSub device. The optimisation parameter is the Levelised Cost of Energy (LCOE) rather than simply total power output. Important optimized design parameters such as the number of floats will be obtained through this research that will help the device to be competitive in the economic market. Different optimisation techniques have been used for this purpose. Two Design of Experiment methods (Taguchi and the Latin hypercube) are used for sensitivity study of the design parameters. Then the genetic algorithm is used for the refinement of the optimum found. The genetic algorithm has been chosen because it is a global optimisation algorithm, it is a free gradient algorithm and it can be used in a parallel computation. In fact, the global optimum is of most interest than local optimum while the gradient of the objective function is not available easily. The parallel computation, instead, decreases the computation time and it is so very useful. This work is limited to a small number of floats as the computational time increases significantly with a larger number of hydrodynamic bodies of the BEM. More specifically, the optimisation carried on considers multi-float configurations of the WaveSub with a number of floats between 1 and 6 . Post-processing of the results will show that an increase of number of floats between 1 and 3 determines a decrease of the LCOE while after that a further larger number of floats contributes only a slight improvement. 


\section{Material and methods}

\subsection{Methodology}

Hydrodynamic independence between the different devices is assumed for the optimisation study of the WaveSub farm. This is a reasonable assumption for small to medium sized arrays if the distance between WECs is large enough. In fact, float spacing between the WECs in the array is assumed to be large enough compared to the wavelength. However, hydrodynamic interactions between the components of the multi-float configuration of each device are taken in account because their distance is smaller. Float spacing between the WECs will be considered in future work where also the layout of the WEC array is considered.

The numerical model of the device is based on Nemoh (Babarit, 2017) and WEC-Sim (Yu et al., 2020). A description of the theory related to these software is described in (Faraggiana et al., 2020). Nemoh is first used to calculate the hydrodynamic coefficients of the WEC that are afterwards used as an input for the time domain simulation in WEC-Sim.

The design parameters considered in the optimisation are the float spacing and number of floats, the float-reactor separation, stiffness, damping and rated power of the PTOs. Some of them require a simulation in Nemoh for every variation (float spacing, float-reactor separation and number of floats) while for others, only a WEC-Sim simulation is necessary. During the optimisation the parameters are considered in the following range:

- Float spacing (From center to center between the floats as a function of the radius of the float): [ $6 \mathrm{R}-25 \mathrm{R}] \mathrm{m}$

- Float number: [1-6]

- Float-reactor separation (Between the centres of the float and of the reactor): [20-84.6] m

- $\quad$ PTO stiffness (kPTO): [5-1000] kN/m

- $\quad$ PTO damping (cPTO): [50-1000] kN/(sm)

- Device rated power: [0-100] MW

The float spacing is investigated from the minimum feasible distance to around four times this minimum. The minimum feasible distance is determined considering the distance between the attachment points of the PTO lines on the reactor. More specifically, these attachment points are described by the position of the pulleys of the PTO lines on the reactor. The float number has been limited to 6 due to the significant increase in the computational cost with increasing number of floats. The float-reactor separation is investigated in a reasonable range considering the sea depth, the size of both the float and the reactor and the design fixed depth of the float. The PTOs are described by a simple translational PTO but a more complex PTO model will be necessary in a future further analysis to follow more accurately the detailed design of a hydraulic PTO. Range of PTO damping and stiffness are set up 
based on the experience of MPS. Finally, the rated power is also one of the parameters to be optimised. At each optimisation iteration, a power matrix is calculated, this gives details of the power produced by the device for each sea state. It is cost efficient to limit the power generated for any one sea state to the rated power. A larger rated power will increase the power produced for the most energetic sea states but the cost of the PTO will be also higher. The specific value of the maximum cap on rated power produced by every sea state does not need to be calculated as part of the time domain WEC-Sim simulation and can be calculated in a post-processing step.

A similar previous optimisation methodology has been explained in (Faraggiana, Masters and Chapman, 2018). The work presented here extends the methodology to account for the post-processing optimisation of the rated power and the LCOE for each time domain simulation. The optimisation scheme for a WaveSub array is shown in Figure 2. The range of the design parameters to investigate is the input of the optimisation while as a result the best combination of design parameters that minimises the LCOE is obtained. These design parameters are the float spacing and number, the float-reactor separation and the stiffness and damping of the PTO. The rated power is considered in a second optimisation (See OPT block of Figure 3) that minimises the LCOE for each design parameters configuration defined by the main optimisation shown in Figure 2. This second optimisation again uses the genetic algorithm. The central block of Figure 2 shows the optimisation process based on a genetic algorithm and on a parallel computation. The genetic algorithm determines the update of the design parameters at each generation to values that minimize the LCOE. The energy calculation is given by the solver that involves the use both of Nemoh and WEC-Sim and the use of a scatter matrix.

It should be noted that multiple simulations are required to compute the optimisation loop, and that this requires a complete time domain wave power calculation for each iteration. This is a significant computational overhead but this can be computed using HPC facilities and parallel computing thanks to the MATLAB function "parfor" (MATLAB, 2019b). This function enables to a model to execute in parallel for cycles in a parallel pool. It is possible to set-up the number of workers that the user wants to use within a multi-processor compute cluster. The optimisation process has been performed six independent times, each one for a different number of floats. This reduces the number of variables for each optimisation and helps to improve computational efficiency and throughput for the parallel computations, in this case, all six optimisations can be started in parallel, even though larger float numbers take a longer time. 
157 Two different Design of Experiment (DOE) approaches have been used for a first stage exploration of the domain. The Taguchi

design can be used to build orthogonal arrays that can help to understand the average response of each design variable on the response (Roy, 2010). It is a fractional factorial design and so the interaction effects between the parameters can't be understood completely. Especially a highly non-linear model is difficult to describe well using this kind of sampling. Ortoghonal arrays have been built based on a MATLAB file exchange (MATLAB, 2019a). The orthogonal array is built based on the paper by (Leung and

Wang, 2001) that establishes a relationship between the number of factors $(N F)$ and levels $\left(Q_{l}\right)$. This relationship can be expressed as

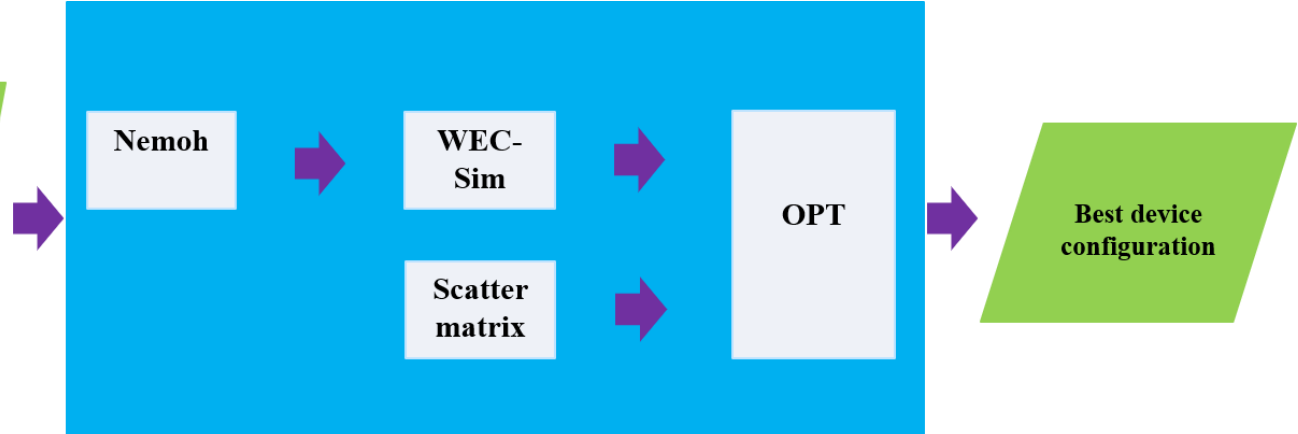

Parallel computation on the Astute 2020 HPC in Swansea University

Optimization

algorithm

Figure 2: The optimisation scheme for a WaveSub array.
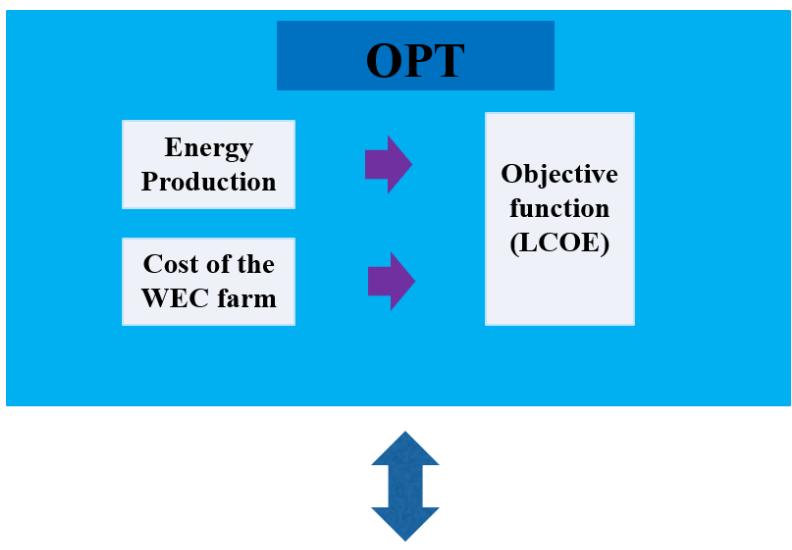

Optimization

algorithm

Figure 3: The post-processing OPT block in detail.

$$
N F=\frac{Q_{l}^{j-1}}{Q_{l}-1}
$$


where $\mathrm{j}$ is an integer number.

A Latin hypercube DOE has also been used for the better equidistribution of the sample points and to account for non-linearities of the response. A surrogate model can be created based on this DOE and it can be used to understand better the relationship between output and input design variables. The model behaviour can be analysed considering the influence on the output of different parameter values. The main goal of this approach is to find a meta-model that is similar to the computationally expensive Nemoh WEC-Sim model.

The Kriging emulator, that is suitable for highly nonlinear responses, has been chosen as surrogate model. In particular, this emulator belongs to the family of linear least squares algorithms. The open-source Oodace toolbox has been used for this purpose (Couckuyt, Dhaene and Demeester, 2014). The estimation of the response variable of a point $\mathrm{x}$ is based on a linear combination of the results of the simulations and it is expressed as

$$
f(x)=\sum_{i=1}^{N} \lambda_{i}(\mathrm{x}) \mathrm{f}\left(\mathrm{x}_{\mathrm{i}}\right)
$$

where $\lambda_{i}$ are the weights obtained through a regression analysis. Then a Gaussian process is built through the residuals.

The Normalised Root Mean Square Error (NRMSE) and the maximum error (MAX) are used to estimate the efficiency of the emulator. They are evaluated for a certain number of testing points $\left(n_{p}\right)$ and are expressed as

$$
N R M S E=\frac{\sqrt{\frac{1}{n_{p}} \sum_{i=1}^{n_{p}}\left(y_{i}-\widehat{y}_{l}\right)^{2}}}{y_{\max }-y_{\min }}
$$

$$
M A X=\max \left(\left|y_{i}-\widehat{y_{l}}\right|\right)
$$

where $y_{i}$ and $\widehat{y}_{l}$ are the real and predicted value of the response.

Once the overall search space has been described, a genetic algorithm has been used as a final stage of the optimisation. The choice of this algorithm was influenced by the fact that information to be able to find the gradient of the objective function is not easily available. It uses different operators such as selection, crossover and mutation that will improve the global fitness at each generation. A more detailed description of the different phases is given here:

- The selection operator is used considering a Stochastic Universal Sampling (SUS).

- A uniform cross-over operator is used. The new population is generated by linear recombination of the previous generation.

- The mutation operator considers a real coded mutation. 
189 An open-source code implemented in MATLAB has been used for the optimisation (Blasco, 2019). This code has been extended 190 to take in account the memory of the previous generations and to account the capability of the surrogate model. In particular the 191 code has been combined with a Kriging surrogate model and its performance has been checked with a very non-linear test function 192 as described in Section 3.2.

\subsection{Computational model set-up}

194 The device considered in the optimisation is the same also used in (Faraggiana et al., 2020).

195 A linear multi-float design is characterized by a long reactor and a series of floats aligned along the wave direction. Figure 4 shows 196 the scheme of the design. The floats are described by a capsule shape with a central cylinder and 2 hemispheres in the sides. The 197 reactor is simplified to a cuboid shape. The mooring connects the reactor and the seabed. The attachment points on the reactor are 198 placed on the corner while the attachment points on the seabed describe the $\mathrm{x}$ and $\mathrm{y}$ spacing as

$$
X_{M S p a c}=\frac{4}{3} R L
$$

$$
Y_{\text {MSpac }}=\frac{7}{4} R W
$$

201 where RL and RW are the length and width dimensions of the reactor. 

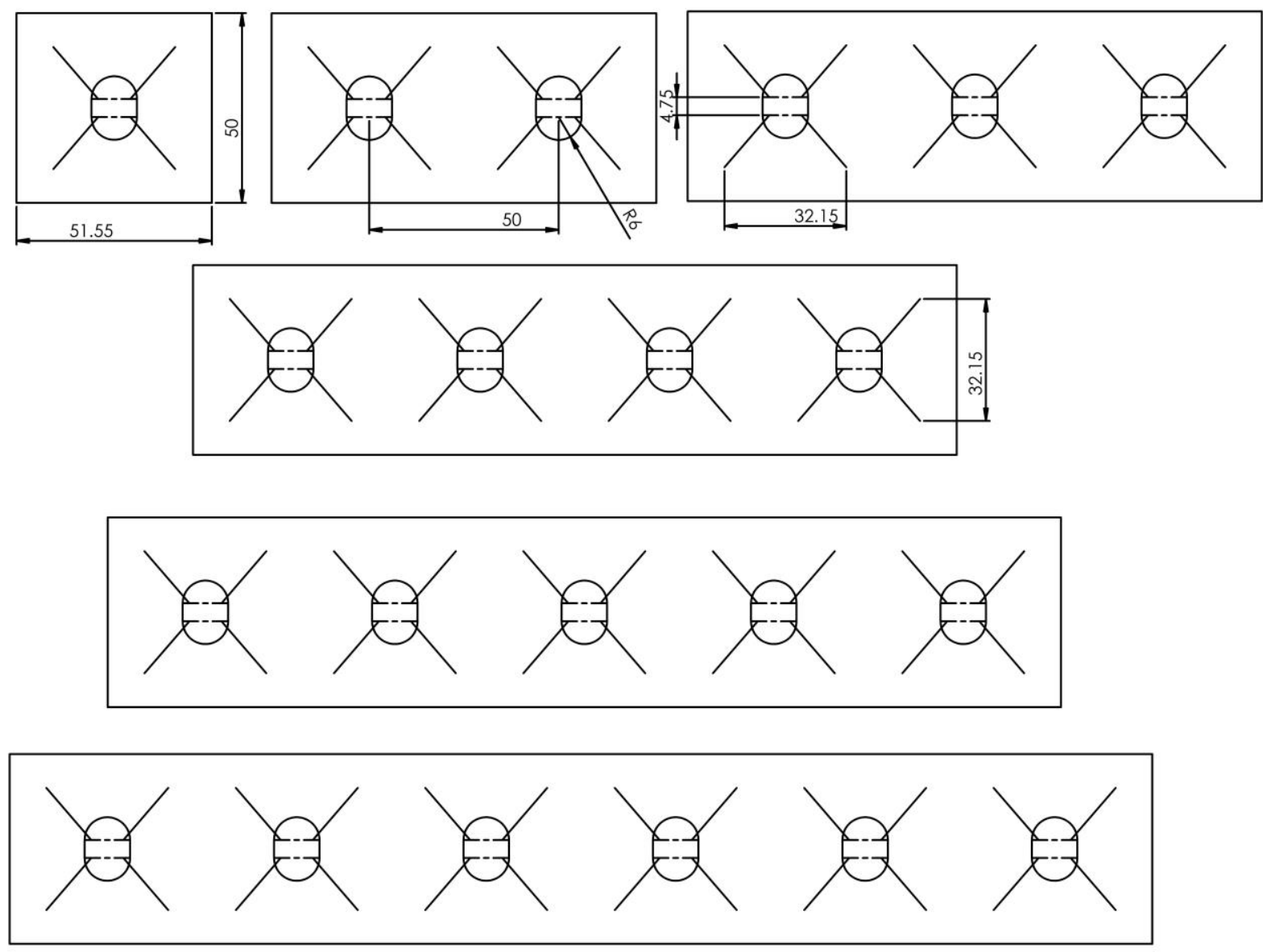

$$
\frac{S N B_{b} \cdot n \text { floats }}{g}=\left(M_{F}-M_{F d}\right) \cdot n_{\text {floats }}+M_{R}-M_{R d b} \cdot \frac{R L}{R L_{b}}
$$

212 where $M_{R}$ is the reactor mass, $S N B_{b}$ is the baseline system net buoyancy, $M_{R d b}$ is the baseline displaced mass of the reactor, $M_{F d}$ 213 is the displaced mass of the float and $M_{F}$ is the mass of the float.

214 The mass of the reactor is the unknown variable of equation (8) and it is finally obtained as

where $R L$ is the reactor length, $R L_{b}$ is the baseline reactor length, $F S$ is the float spacing and $n f l o a t s$ is the number of floats. Existing engineering drawings were used to establish a baseline system to give reference values of the system net buoyancy, reactor displaced mass and reactor length. The system net buoyancy has been designed dependent on the number of floats while the designed displaced mass of the reactor has been obtained using a linear relationship with reactor length. Th system net buoyancy is calculated as

$$
R L=R L_{b}+F S \cdot\left(n_{\text {floats }}-1\right)
$$




$$
M_{R}=\left(\frac{S N B_{b}}{g}+M_{F d}-M_{F}\right) \cdot n_{f l o a t s}+M_{R d b} \cdot \frac{R L}{R L_{b}}
$$

A specific wave farm location has been identified for the optimisation. This was the Berth A wave buoy in Belmullet, the Atlantic Marine Energy Test Site (AMETS), Ireland (Ireland Ocean Energy Expertise, 2019). This area has a water depth of $100 \mathrm{~m}$ which matches the designed depth of the WaveSub device. A wave data analysis for the years from 2013 to 2017 has been used to obtain information about the scatter matrix and the raw spectra. The Incident Energy per metre of wavefront (IE) for each sea state (Holthuijsen, 2010) can be approximated for deep sea water as

$$
I E_{i j}=O_{i j} \cdot 8760 \cdot 0.49 \cdot H_{s i}^{2} T_{e j}
$$

where $O_{i j}$ is the occurrence for a particular sea state.

The full scatter matrix is obtained for significant heights between $0.25 \mathrm{~m}$ and $15.5 \mathrm{~m}$ and for energy periods between $4 \mathrm{~s}$ and $22 \mathrm{~s}$. The spacing considered in the significant height is $0.25 \mathrm{~m}$ while the spacing considered in the energy period is $0.5 \mathrm{~s}$. To reduce computational effort, a reduced scatter matrix that considered three main ranges of energy periods has been used. There is a range of energy periods under $9 \mathrm{~s}$, between $9 \mathrm{~s}$ and $11 \mathrm{~s}$ and over $11 \mathrm{~s}$. The total sum of the occurrences of the scatter matrix is 1 and so the reduced scatter matrix approximates mostly in the lower and upper energy period range. In particular, a sea state in the reduced scatter matrix considers the sum of the occurrences of the various energy periods considered in the full scatter matrix. Note that the optimisation is carried out on energy, not power. The energy matrix that corresponds to the sea states is obtained as a multiplication of the power and occurrences matrices together. It is a good indicator to show where the device should be tuned to obtain the maximum amount of power generated. An optimized device for the most energetic sea states will give the maximum total amount of energy generated during the year. An optimisation of the device for the most frequently occurring sea states will give a more constant power generated but it is likely that the total amount of energy will be less. Maximization of the total energy produced from the device has been preferred in this paper.

The lower and upper range of the energy periods have been reduced to account for only the spectra of the most energetic sea states for this calculation ( $87 \%$ of the total energy of the full scatter matrix). So the device is optimised for the most energetic sea states. The spectra for each of the simulated sea states have been obtained as an average of the spectra in each energy period range (See Figure 5). Peak period and energy period properties for each sea state simulated are reported in Table 1. A comparison between the raw spectra and the theoretical spectra that minimizes the standard deviation between them is made. In particular, a narrower spectra for higher peak periods has been observed. Finally, the spectra used in the simulations has been normalized for a significant height of $1 \mathrm{~m}$. The simulated sea states are just dependent on the wave energy period but not on the significant height (See Table 
243 1). Using the theory of (Cruz, 2007) for linear wave hydrodynamics in deep water, a first approximation of the power produced by 244 the device can be estimated to be dependent on the square of the significant height. Apart from minor drag terms, all the forces 245 considered in the numerical model of the dynamic system are linear. Subsequent to the analysis, the effect of wave height is 246 recovered in a post processing stage.

Table 1: The sea states used in the optimisation.

\begin{tabular}{|l|l|l|l|}
\hline $\begin{array}{l}\text { Approximated } \\
\text { theoretical } \\
\text { spectra }\end{array}$ & Bretschneider & $\begin{array}{l}\text { JONSWAP } \\
(\text { Gamma=1.1) }\end{array}$ & $\begin{array}{l}\text { JONSWAP } \\
(\text { Gamma=1.3) }\end{array}$ \\
\hline $\begin{array}{l}\text { Approximated } \\
\text { range of Te (s) }\end{array}$ & $7<=\mathrm{Te}<=9$ & $9<=\mathrm{Te}<=11$ & $11<=\mathrm{Te}<=13$ \\
\hline $\mathbf{H s}(\mathbf{m})$ & 1 & 1 & 1 \\
\hline $\mathbf{T e}(\mathbf{s})$ & 8.32 & 10.03 & 11.83 \\
\hline $\mathbf{T p}(\mathbf{s})$ & 10 & 11.76 & 14.29 \\
\hline $\mathbf{T p} / \mathrm{Te}$ & 1.20 & 1.17 & 1.21 \\
\hline
\end{tabular}

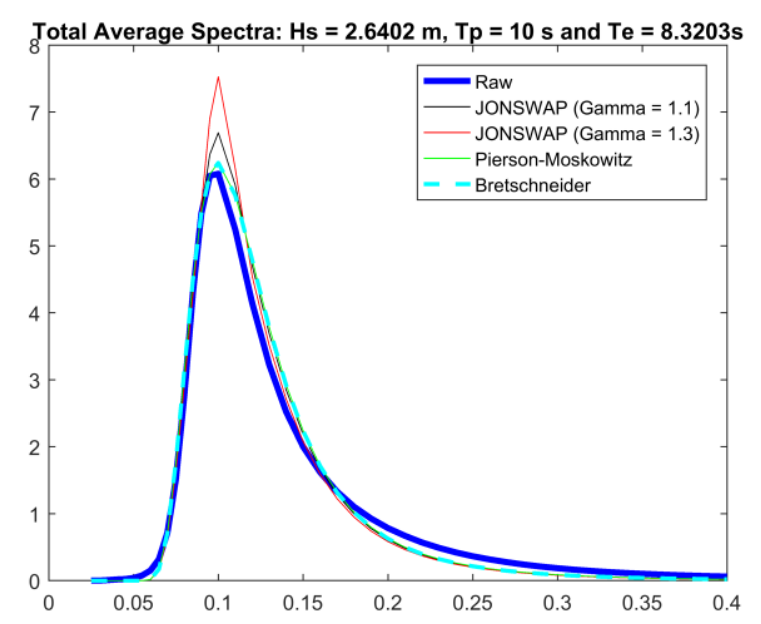




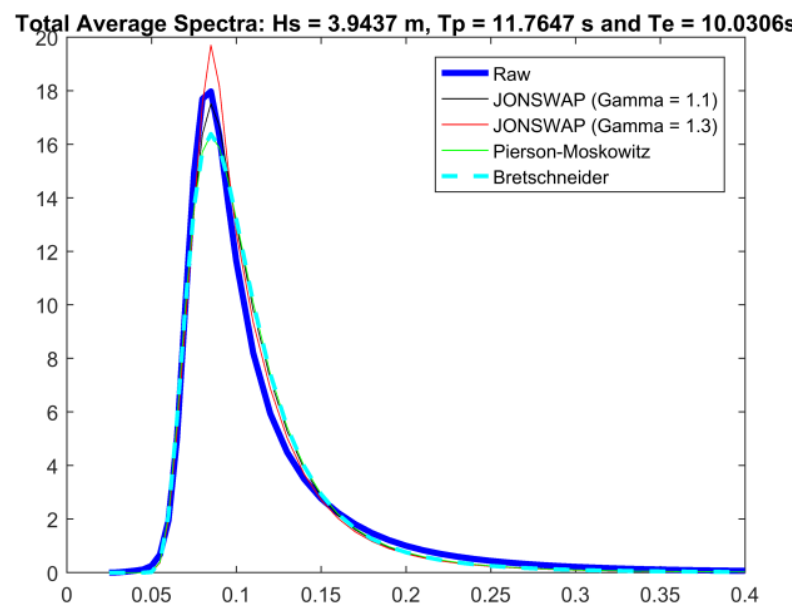

(b)

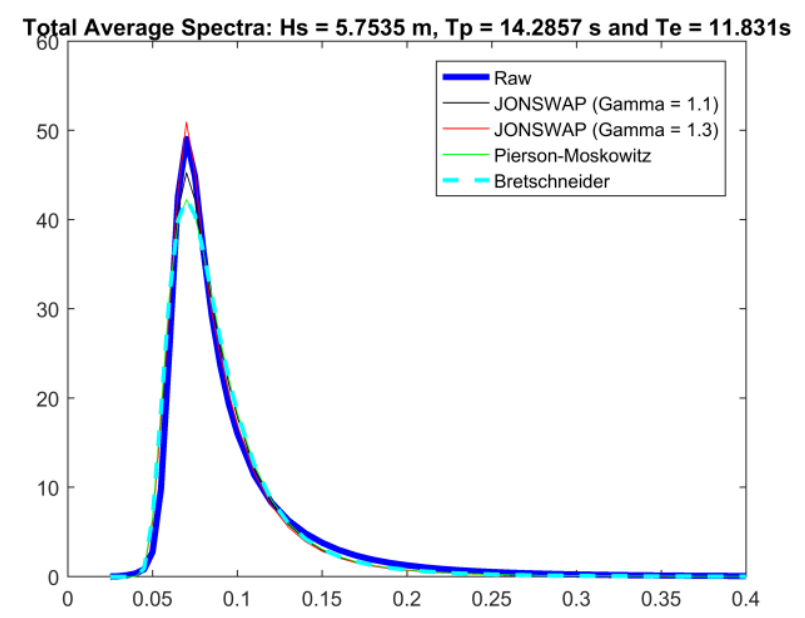

(c)

258 Figure 5: The average spectra between $T e=7$ and $T e=9(a), T e=9$ and $T e=11(b), T e=11$ and Te=13 (c) for the Belmullet wave site between

260 The calculation of the Levelized Cost Of Energy (LCOE) is one of the most critical issues of the optimisation process. A cost 261 estimation is very difficult for Wave Energy Converters because there isn't enough experience in this sector to use known costs 262 from existing farms. However, an existing cost calculation developed by MPS Ltd was used in this work. It was based on the 263 experience of the company and on information given by different manufacturers and components suppliers. Details of the structure 264 of the calculation can be found in (Faraggiana, Masters and Chapman, 2018). A brief description of the CAPEX and OPEX 265 calculation is as follows. The CAPEX is obtained as the sum of various components and it can be described as

$$
\begin{gathered}
\text { CAPEX }=\left(\text { Cost }_{\text {Structure }}+\text { Cost }_{\text {PTO }}+\text { Cost }_{\text {Control }}+\text { Cost }_{\text {Grid }}+\text { Cost }_{\text {Mooring }}+\text { Cost }_{\text {Installation }}\right. \\
\left.+ \text { Cost }_{\text {Margin }}\right) \cdot N_{\text {devices }}
\end{gathered}
$$


Where Cost $_{\text {Structure }}$ is the material cost of the floats and the reactor, Cost $_{\text {PTO }}$ is the cost of the hydraulic PTO where the secondary system depends on the rated power, Cost $_{\text {Control }}$ is the control cost of the PTO, Cost $_{\text {Grid }}$ is the grid connection cost, Cost $_{\text {Mooring }}$ is the mooring cost, Cost $_{\text {Installation }}$ is the installation cost, Cost $_{\text {Margin }}$ is the margin cost and $N_{\text {devices }}$ is the number of devices. When considering the cost of a single device, most of the costs have a fixed element, that is not dependent on the configuration, and a variable cost that changes with the optimisation. The optimisation considers a WEC farm of 400MW and so the number of devices changes, particularly with the number of floats. Consequently, the total wave farm costs of the control, grid, mooring, installation and margin are reduced for larger numbers of floats per device.

The main simulation parameters for Nemoh and WEC-Sim are shown in Table 2. The ramp time is used for the calculation of the excitation force at the beginning of the numerical simulation to have a gradual increase of the flow until the full wave conditions are reached (Yu et al., 2020). From previous experience, the number of wave frequencies and the duration of the simulation time are assumed to give a reasonable accuracy on the results. Interpolation of the hydrodynamic coefficients from Nemoh is used to obtain their values for further frequencies. The time simulation is between 15-30 minutes, the typical record length suggested for irregular waves (Holthuijsen, 2010).

\begin{tabular}{|l|l|}
\hline \multicolumn{2}{|c|}{ Simulation parameters } \\
\hline Water depth (m) & 100 \\
\hline Density sea water $\left(\mathbf{k g} / \mathbf{m}^{\mathbf{3}}\right)$ & 1026 \\
\hline Minimum frequency (Hz) & 0.05 \\
\hline Maximum frequency (Hz) & 5 \\
\hline Number of frequencies (-) & 51 \\
\hline Simulation time (s) & 1300 \\
\hline Ramp time (s) & 100 \\
\hline Solver WEC-Sim & Ode45 \\
\hline Timestep (s) & 0.02 \\
\hline
\end{tabular}

283 The total simulation time for the calculation of the energy production for a specific settings of the design parameters is determined mainly by the simulation time of Nemoh and WEC-Sim (around $1 \mathrm{~h}$ for WEC-Sim while at least $3 \mathrm{~h}$ for Nemoh for the largest 
number of floats configuration and the fine mesh). More specifically, Nemoh simulation time depends on the float spacing because there will be a larger number of panels of the reactor for a larger float spacing. WEC-Sim simulation time cannot be reduced because of the needed time domain simulation. The mesh of the float and of the reactor, instead, has been obtained considering a balance between two main factors: the computational time and the accuracy of the results. The optimisation requires the minimum number as possible of panels of the mesh to have a larger number of simulations. By contrast accuracy requires a fine mesh. (Faraggiana et al., 2020) shows the mesh independence from a coarser mesh to a finer mesh. The hydrodynamic coefficients in the main degrees of freedom (surge, heave and pitch) are almost identical between the different meshes. So a coarse mesh could be used for the optimisation to obtain a reasonable accuracy with a simulation reduction time at least 4 times less compared to the fine mesh case. Specifically, a quadrangular mesh has been used for the float and the reactor. The mesh of the float used a similar amount of nodes as the tank testing case (80). The mesh considers quadrangular elements with 81 panels. The mesh of the reactor has been initially designed for a length equal to the tank testing size of $51.55 \mathrm{~m}$ (98 panels). When the reactor changes size in the optimisation, the corresponding mesh has been obtained using a linear scaling of this mesh. In this way the number of panels of the reactor is significantly reduced with a computational time remarkably decreased especially for large float spacing and number of floats. The linear scaling of the mesh of the reactor probably will not create a good element aspect ratio. However, the main motion is expected to come from the floats and so the accuracy of the hydrodynamics of the reactor is of secondary importance. In fact, a steady reactor described by a high mooring stiffness is part of the design process of the device and so small motions of the reactor are expected and a higher error in this movement is acceptable. The optimisation will consider the power produced from the device that depends on the relative motion between the float and the reactor. The mesh convergence of the reactor for a 6 floats device and a float spacing of 50m is shown in Figure 6. The coarse mesh (96 panels) is obtained applying the scaling for the reactor length while the moderate (165 panels) and the fine mesh (335 panels) are obtained directly from Salome (Codeaster, 2019) for the exact length of the reactor. The main motions of the reactor are in surge and heave and generally the coarse mesh approximates quite well compared to the fine mesh results.
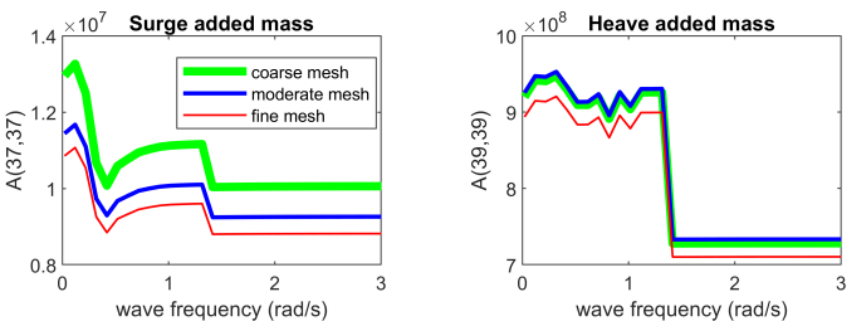


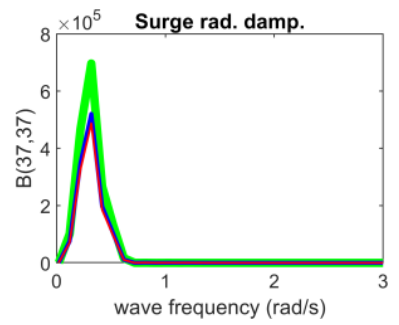

(c)

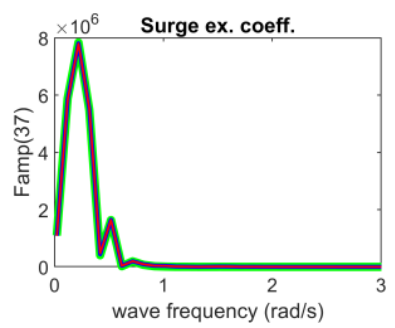

(e)

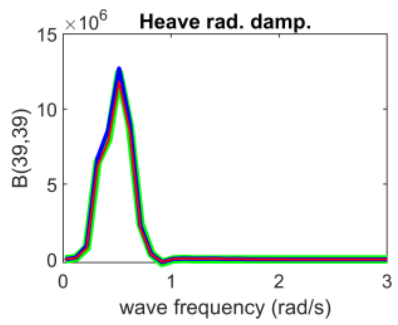

(d)

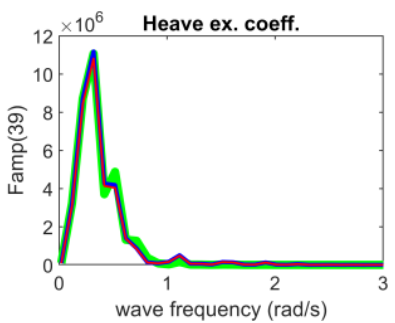

(f)

Figure 6: Surge and heave added mass coefficients of the reactor (a,b), surge and heave radiation damping coefficients of the reactor (c,d), surge and heave excitation coefficients of the reactor (e,f). The simulation is made for a 6 float configuration and a float spacing of $50 \mathrm{~m}$.

The hydrodynamic coefficients don't consider the drag generated by the floats and the reactor. For this reason a drag term has been added to the time domain simulation in WEC-Sim in a similar way as described in (Faraggiana et al., 2020). In this research experimental and numerical data for different regular waves of a 3 float multi-float configuration were analysed. The drag term is described as

$$
\vec{D}=\frac{1}{2} C_{d} \rho A \vec{v}|\vec{v}|
$$

Where $A$ is the characteristic area of the body, $v$ is the body velocity, $C_{d}$ is the drag coefficient, $D$ is the drag force and $\rho$ is the density. Through a comparison of experimental and numerical data, the paper suggests a drag coefficient of 0.7 for the floats and that is used in this work. The reactor is simplified as a cuboid shape and it is very large compared to the wavelength and so a small drag coefficient is considered in a first approximation (0.5).

\section{Results}

\subsection{Taguchi and Latin hypercube DOE}

The Taguchi optimisation is an efficient way to have a first estimate of the optimum with a lower number of simulation cases and can also be used for sensitivity analysis purposes. The Taguchi optimisation gives an estimation of the average influence of each parameter on the response but neglecting interaction effects between the variables. The Latin hypercube can be used for the same 
purpose and it is designed to have an equidistribution of the sample points and can consider interaction effects. The Latin hypercube is also used for a sensitivity study generating a surrogate Kriging model of the sample points simulated. The number of simulations is limited to 100 for computational reasons. The Taguchi DOE is obtained for a Taguchi orthogonal array with 10 levels (Roy, 2010).

Figure 7 shows the results of the sensitivity analysis of the Taguchi DOE. The design parameters are investigated with 10 values within the range defined in the methodology section. More specifically, the 10 values are equidistributed between the minimum and maximum values of each range of the design parameters. The $\mathrm{x}$ axis shows the 10 values investigated for each design parameter while the $y$ axis describes the normalized LCOE for that particular optimisation of number of floats. The LCOE described in this Figure for each design parameter has been also averaged by the influence of the other parameters. The optimal LCOE for each configuration is obtained by the combinations of design parameters that give the lowest LCOE. Figure 7 shows that the average LCOE for each design parameter is very similar between the multi-float configurations. Main difference between them is the absolute LCOE value as demonstrated also in Table 3 in which the optimal LCOE decreases with an increased number of floats. The float-reactor separation gives a minimum LCOE in the central range. Optimal float spacing is given by the lowest value of the range considered for all the optimisation cases. There is also a local optimum for a larger float spacing but this one is not worth investigating despite the larger energy produced because of the feasibility uncertainties of such long reactor. PTO stiffness and damping coefficients have a different influence on the minimum LCOE. The best PTO stiffness coefficient is in the lower area of the range considered while the best PTO damping coefficient is in the higher area. The value of each parameter that gives the minimum LCOE from the Taguchi analysis for each WaveSub configuration are shown in Table 3. The model has been re-run with the suggested optimal values of each design parameter and as expected the multi-float configurations have given a better optimum compared with the runs in the original Taguchi array. Similar optimal LCOE are obtained for the 5 and 6 float configurations. This suggests that if a large number of floats are included in one device, this will not improve the LCOE any further.

Figure 8 shows the variation of total energy produced and the capacity factor with varying numbers of floats. The capacity factor is around 0.42 for all of the multi-float configurations and it increases slightly as the number of floats increases. The energy produced from the device shows a proportional increase with the number of floats. The LCOE is shown in Figure 9 and this decreases with number of floats, mainly because of the decrease of the largest cost, CAPEX. Looking more closely, increasing the number of floats per device decreases the total number of devices in the array and therefore reduces the control and instrumentation, installation, grid connection, mooring and margin costs (See Table 3). Structure and PTO costs show a similar total cost for each float configuration. Structural costs are similar between the configurations because this cost is related to size and the optimal float 
spacing is similar for all configurations. The total farm rated power is constant and so the total PTO cost is similar. Small differences in the total PTO costs are due to the finite number of devices with a total rated power sum that is not exactly $400 \mathrm{MW}$.

354 OPEX and decomissioning costs are decreasing with an increase of the number of floats per device due to a smaller number of 355 devices. These costs are small compared to the CAPEX and so their influence on the LCOE is less significant. Optimal results of the Latin hypercube DOE are shown in Table 4. It is interesting to notice that the float spacing converges towards two different values, the lower one because of lower costs and the higher value such for the 3 float configuration because the device produces more energy when the float spacing is similar to the wavelength (Budal, 1977). However, irregular waves have an averaging effect of the constructive interactions between the floats and so other factors such as the interaction effects between the design parameters could have played an important role. The capacity factor increases for the multi-float configurations and becomes very similar between 4 and 6 multi-float configurations as shown in Figure 8. Figure 9 shows that the Latin hypercube optimisation has the same trends as the Taguchi with decreasing LCOE for the larger multi-float configurations mainly due to decreasing CAPEX.

364 Figure 10 shows a 2D surface representation of the influence of the optimal stiffness and damping of the PTO on the LCOE for 365 the Latin hypercube DOE and a 6 multi-float configuration. In-fact this configuration is considered one of the most promising 366 between the ones analysed. PTO stiffness and damping are investigated while the other two are fixed to the optimal values found 367 by the Latin hypercube. The darker blue of the plots shows where the optimal (lower) LCOE can be found. The conclusions of this 368 graph (Figure 10) agree with the Taguchi method for the average response of the design parameters (Figure 7). Optimal values are 369 within the lower range for PTO stiffness, and generally larger values for the PTO damping. It is observed that LCOE increases 370 rapidly for lower values of the PTO damping. The Normalised Root Mean Square Error (NRMSE) and the maximum error (MAX) 371 are used to estimate the efficiency of the surrogate model and they are calculated following (González-Gorbeña, Qassim and 372 Rosman, 2018). The NRMSE and the maximum error are shown at the bottom of Table 4 . The NRMSE is always under 0.1 and so as reported in (González-Gorbeña, Qassim and Rosman, 2018) the surrogate model has reasonable predictive capabilities. 


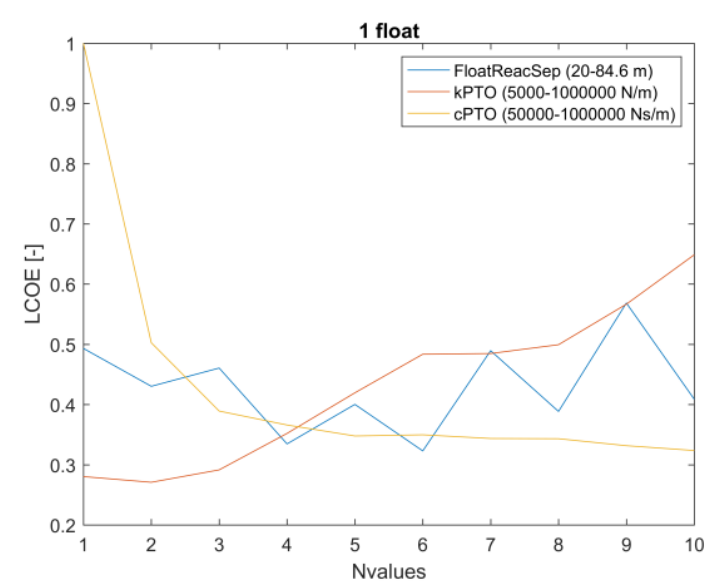

(a)

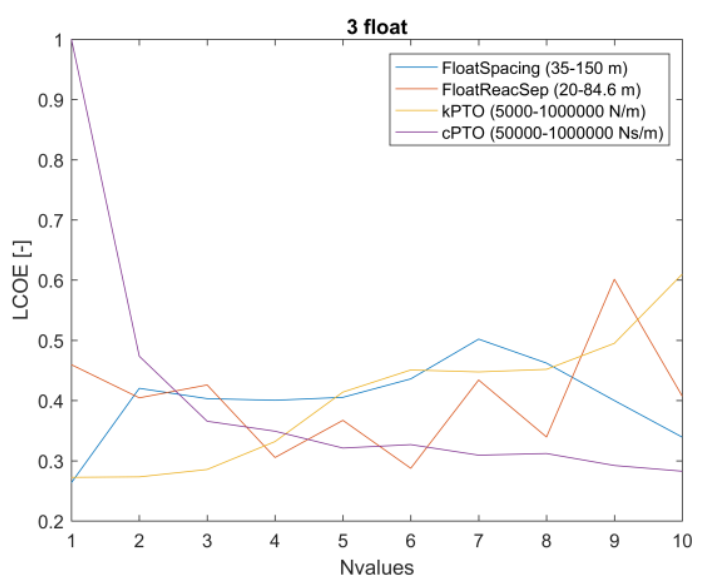

(c)

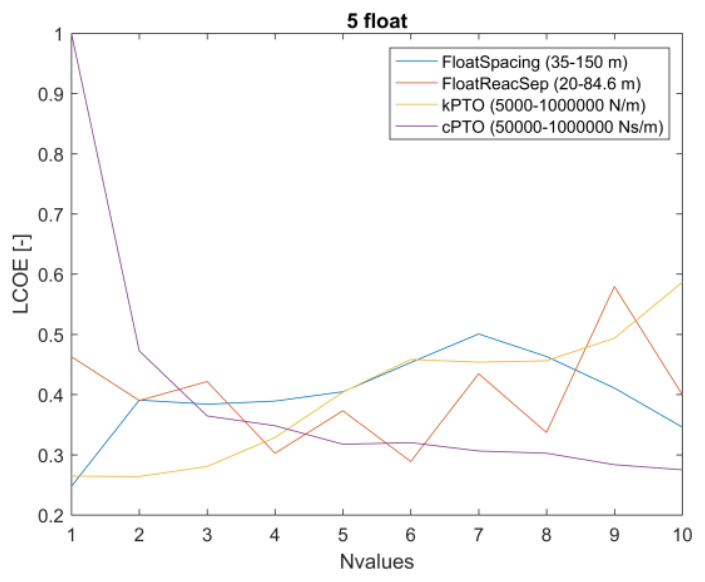

(e)

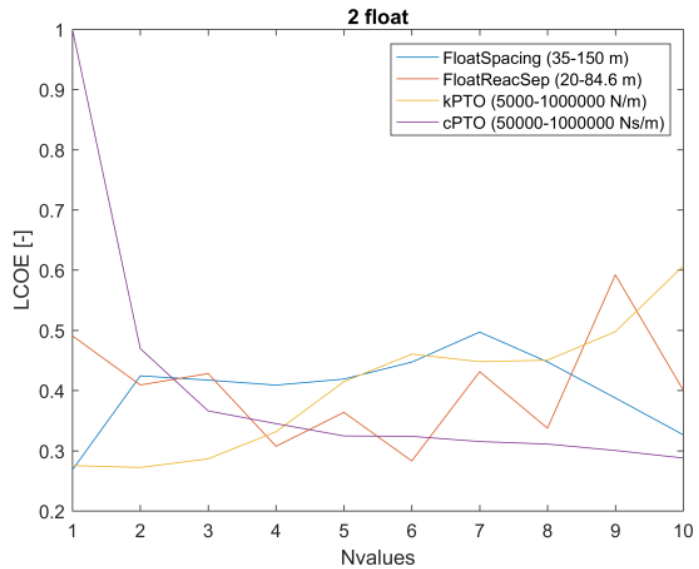

(b)

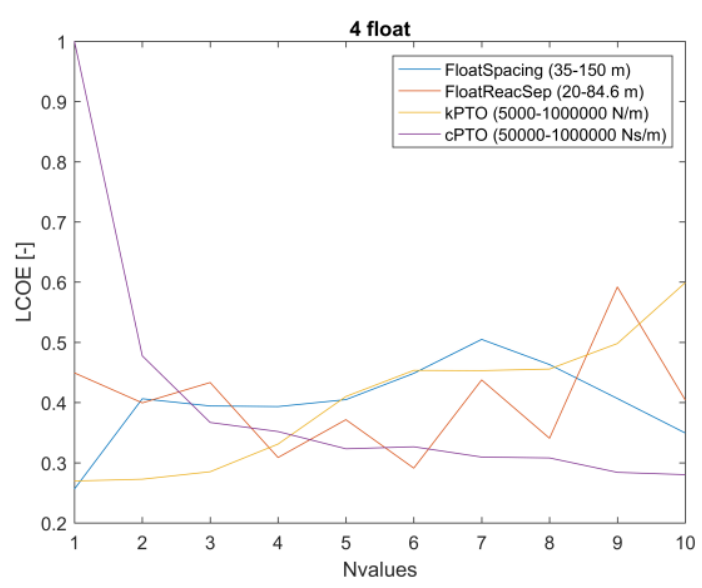

(d)

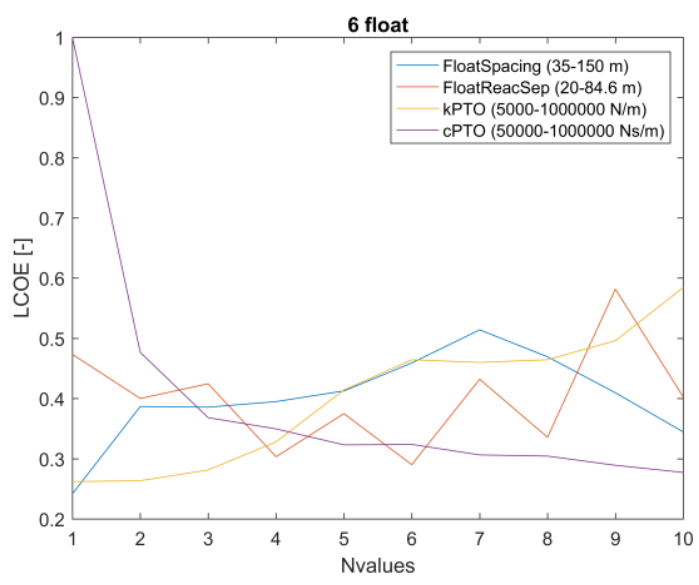

(f)

374 Figure 7: The normalized LCOE sensitivity of the various design parameters from the Taguchi DOE for 1 float (a), 2 floats (b). 3 floats (c), 4 


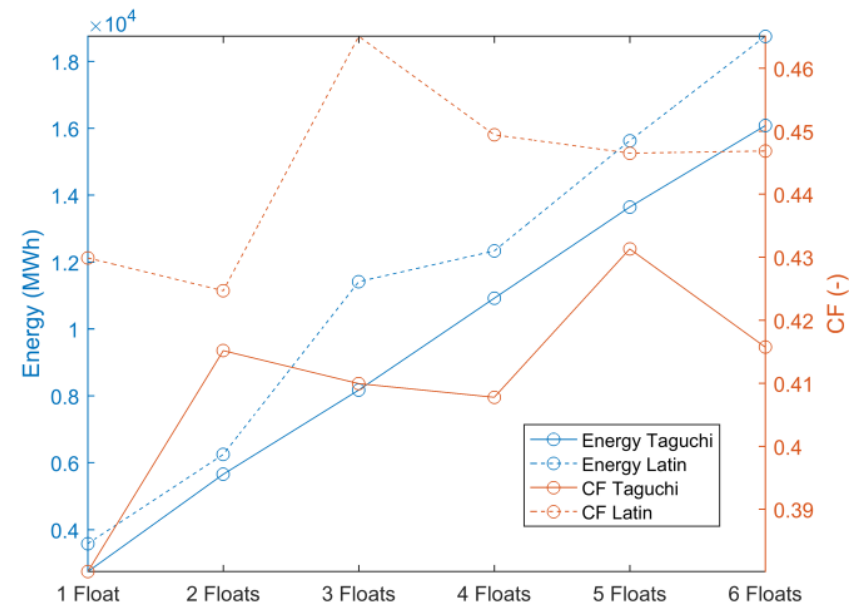

Figure 8: The energy produced and the capacity factor for the optimal cases given Table 5by the Taguchi and Latin hypercube DOE.

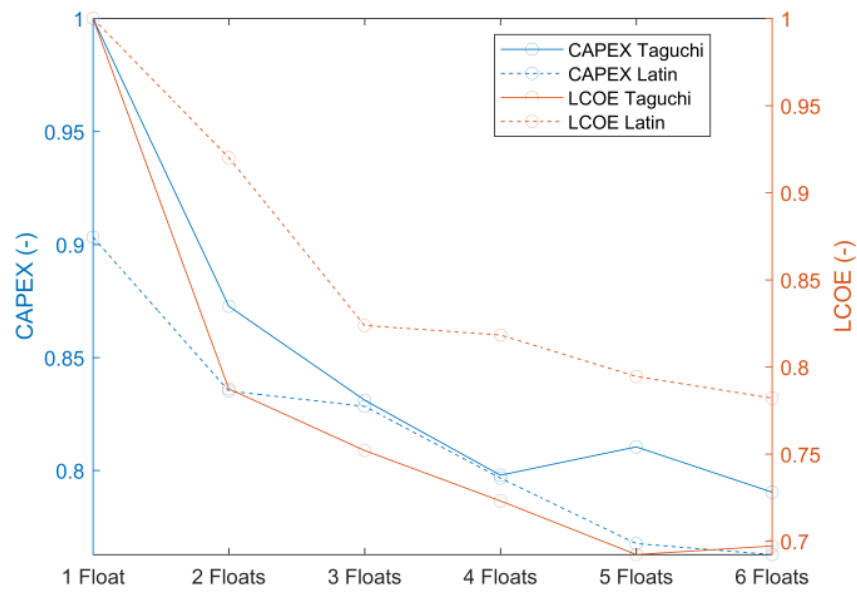

Figure 9: The normalized CAPEX and the LCOE for the optimal cases given by the Taguchi and Latin hypercube DOE.

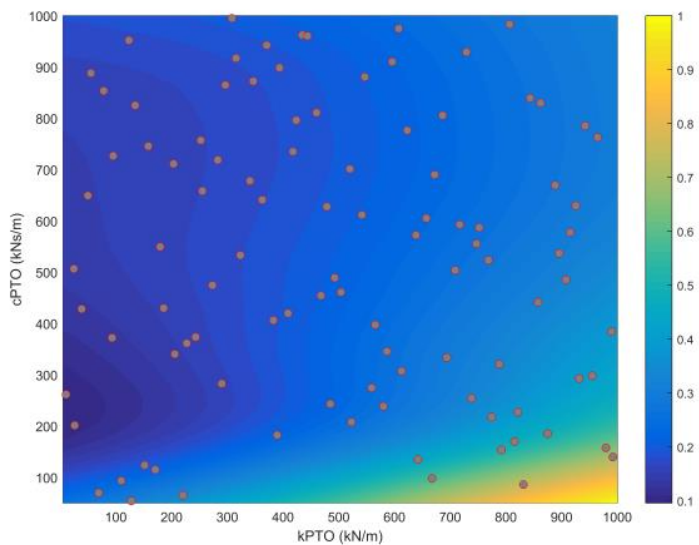

382 Figure 10: The surrogate model for the optimal case of the Latin hypercube DOE for a 6 float configuration. PTO stiffness and damping are investigated while the other 2 parameters are fixed to the optimal case. Darker blue shows where the optimal values of the LCOE are expected. 
Table 3: The design parameters and main results for each multi-float WaveSub configuration derived for the optimum obtained from the

Taguchi DOE. The LCOE and the CAPEX have been normalized to the maximum value obtained.

\begin{tabular}{|l|l|l|l|l|l|l|}
\hline Number of floats & $\mathbf{1}$ & $\mathbf{2}$ & $\mathbf{3}$ & $\mathbf{4}$ & $\mathbf{5}$ & $\mathbf{6}$ \\
\hline Float spacing (m) & - & 35 & 35 & 35 & 35 & 35 \\
\hline Float-reactor Sep (m) & 56 & 56 & 56 & 56 & 56 & 56 \\
\hline kPTO (kN/m) & 116 & 116 & 5 & 5 & 116 & 5 \\
\hline cPTO (kNs/m) & 1000 & 1000 & 1000 & 1000 & 1000 & 1000 \\
\hline RatedP (MW) & 0.8 & 1.6 & 2.3 & 3.1 & 3.6 & 4.4 \\
\hline Energy device (GWh/y) & 2.7 & 5.7 & 8.2 & 10.9 & 13.6 & 16.1 \\
\hline Ndevices & 485 & 257 & 176 & 131 & 111 & 91 \\
\hline CF & 0.38 & 0.42 & 0.41 & 0.41 & 0.43 & 0.42 \\
\hline CAPEX (-) & 1.000 & 0.873 & 0.831 & 0.798 & 0.810 & 0.790 \\
\hline CAPEX-Structure (-) & 0.455 & 0.452 & 0.454 & 0.446 & 0.469 & 0.459 \\
\hline CAPEX-PTO (-) & 0.278 & 0.277 & 0.278 & 0.278 & 0.278 & 0.279 \\
\hline CAPEX-Control (-) & 0.055 & 0.029 & 0.020 & 0.015 & 0.013 & 0.010 \\
\hline CAPEX-Grid (-) & 0.054 & 0.029 & 0.020 & 0.015 & 0.013 & 0.011 \\
\hline CAPEX-Mooring (-) & 0.037 & 0.020 & 0.013 & 0.010 & 0.008 & 0.007 \\
\hline CAPEX-Installation (-) & 0.012 & 0.007 & 0.005 & 0.004 & 0.004 & 0.004 \\
\hline CAPEX-Margin (-) & 0.110 & 0.058 & 0.040 & 0.030 & 0.025 & 0.021 \\
\hline OPEX (y-1) & 0.008 & 0.005 & 0.004 & 0.003 & 0.003 & 0.003 \\
\hline Decommissioning (-) & 0.005 & 0.003 & 0.002 & 0.001 & 0.001 & 0.001 \\
\hline LCOE (-) & 1.00 & 0.79 & 0.75 & 0.72 & 0.69 & 0.70 \\
\hline
\end{tabular}

387 Table 4: The design parameters and main results for each multi-float WaveSub configuration derived for the optimum obtained from the Latin 388 hypercube DOE. The LCOE and the CAPEX have been normalized to the maximum value obtained.

\begin{tabular}{|l|l|l|l|l|l|l|}
\hline Number of floats & $\mathbf{1}$ & $\mathbf{2}$ & $\mathbf{3}$ & $\mathbf{4}$ & $\mathbf{5}$ & $\mathbf{6}$ \\
\hline Float spacing (m) & - & 40 & 101 & 40 & 40 & 40 \\
\hline Float-reactor Sep (m) & 42 & 35 & 21 & 35 & 35 & 35 \\
\hline kPTO (kN/m) & 110 & 25 & 11 & 25 & 25 & 25 \\
\hline cPTO (kNs/m) & 94 & 507 & 262 & 507 & 507 & 507 \\
\hline RatedP (MW) & 1.0 & 1.7 & 2.8 & 3.1 & 4.0 & 4.8 \\
\hline Energy device (GWh/y) & 3.6 & 6.3 & 11.4 & 12.3 & 15.6 & 18.8 \\
\hline Ndevices & 420 & 238 & 143 & 128 & 100 & 84 \\
\hline CF & 0.43 & 0.42 & 0.47 & 0.45 & 0.45 & 0.45 \\
\hline
\end{tabular}




\begin{tabular}{|l|c|c|c|c|c|c|}
\hline CAPEX (-) & 1.000 & 0.925 & 0.917 & 0.882 & 0.850 & 0.844 \\
\hline CAPEX-Structure (-) & 0.436 & 0.471 & 0.520 & 0.494 & 0.480 & 0.482 \\
\hline CAPEX-PTO (-) & 0.307 & 0.307 & 0.307 & 0.308 & 0.307 & 0.309 \\
\hline CAPEX-Control (-) & 0.053 & 0.030 & 0.018 & 0.016 & 0.013 & 0.011 \\
\hline CAPEX-Grid (-) & 0.052 & 0.030 & 0.018 & 0.017 & 0.013 & 0.011 \\
\hline CAPEX-Mooring (-) & 0.035 & 0.020 & 0.012 & 0.011 & 0.008 & 0.007 \\
\hline CAPEX-Installation (-) & 0.011 & 0.007 & 0.005 & 0.005 & 0.004 & 0.004 \\
\hline CAPEX-Margin (-) & 0.106 & 0.060 & 0.036 & 0.032 & 0.025 & 0.021 \\
\hline OPEX (y $\left.{ }^{-1}\right)$ & 0.008 & 0.005 & 0.004 & 0.004 & 0.004 & 0.004 \\
\hline Decommissioning (-) & 0.005 & 0.003 & 0.002 & 0.002 & 0.001 & 0.001 \\
\hline LCOE (-) & 1.00 & 0.92 & 0.82 & 0.82 & 0.79 & 0.78 \\
\hline NRMSE (-) & 0.03 & 0.05 & 0.08 & 0.08 & 0.06 & 0.05 \\
\hline MAX (-) & 1.00 & 0.77 & 1.17 & 1.37 & 0.76 & 0.77 \\
\hline
\end{tabular}

\subsection{The investigation of the genetic algorithm performance}

391 The performance of the genetic algorithm has been investigated applying various modifications. The memory of the previous evaluations is accounted for in the algorithm as well as the tuning of the mutation, cross-over coefficients and of the population and the capability of the surrogate model. The sample points already evaluated have been changed with new ones near the global optimum obtained. In particular, the new points have been randomized around the global optimum and their distance from it has been decreased using a cubic relationship by generation in generation. This can be expressed as

$$
x d_{\text {New Point }}=x d_{\text {min }}+\frac{\frac{x d_{\text {range }}}{2} \cdot \text { rand }}{g e n^{3}}
$$

where $x d_{\min }$ represents the combination of design parameters that minimize the objective function, $x d_{\text {range }}$ is the search space of the design variables, rand is a random vector with the same dimension of the design variables and gen is the number of generations. Finally if the evaluation of this new point is worse than the old one, then the old one will be restored. No specific references were used for this change.

401 in generation. This approach was inspired by the adaptive genetic algorithm (Mahmoodabadi and Nemati, 2016). The particle 402 swarm approach has been considered for this purpose but applied to a single particle that means that the best and the global position 403 of the particle are coincident. The particle is described by 3 elements: the mutation probability, the cross-over probability and the 404 population. The particle swarm algorithm has been obtained from (Yarpiz, 2019). The method is expressed as following: 
- If the optimum found or the maximum of the generation relative to the previous one is decreased then the particle swarm algorithm is used.

- If the previous condition is not verified then the new particle is generated randomly.

The population keeps the best individuals if the population is reduced while it considers new random individuals if the population is increased.

The open-source genetic algorithm has been improved considering also the optimisation capability of the surrogate model. A Kriging surrogate model (Couckuyt, Dhaene and Demeester, 2014) has been adopted for its high efficiency for non-linear responses. This model is updated by generation in generation with the simulation carried out by the genetic algorithm. The surrogate model is introduced in the genetic algorithm through the use of an elitism of 10\% the number of population. The surrogate model is evaluated for new random points with a number of points equal to the same number of evaluations computed until that moment by the genetic algorithm. It is therefore supposed that the efficiency of the surrogate model will increase with the number of evaluations of the genetic algorithm. Then the best individuals evaluated by the surrogate model are added in the next generation. The huge advantage of the surrogate model is that enables the user to estimate results at a very low computational time compared to the real simulation.

These changes have brought an improvement in the test function of the Schwefel Function example case with 2 variables (See Figure 11). This function has many local minima and so the convergence to the global minimum is more difficult. In Figure $11 \mathrm{~b}$ the objective function has been obtained as an average of 1000 different optimisations. The genetic algorithm without the tuning consider a cross-over coefficient of 0.9 , mutation coefficient of 0.1 and a population of 100 individuals while the genetic algorithm with the tuning considers a range of variation between $0-1$ of the cross-over coefficient, $0-0.3$ of the mutation coefficient and 50150 of the number of individuals.

The algorithm that considers all the improvements shows the highest efficiency. However these optimisations that were including the Kriging surrogate model were limited to 11 generations because the simulation time, required by the Kriging surrogate model to fit over around 1000 simulation data points, becomes too high. 


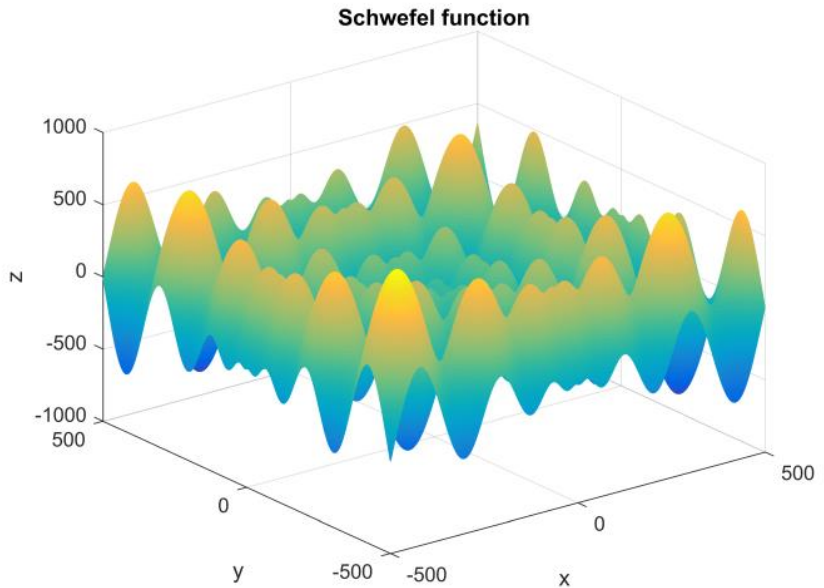

(a)

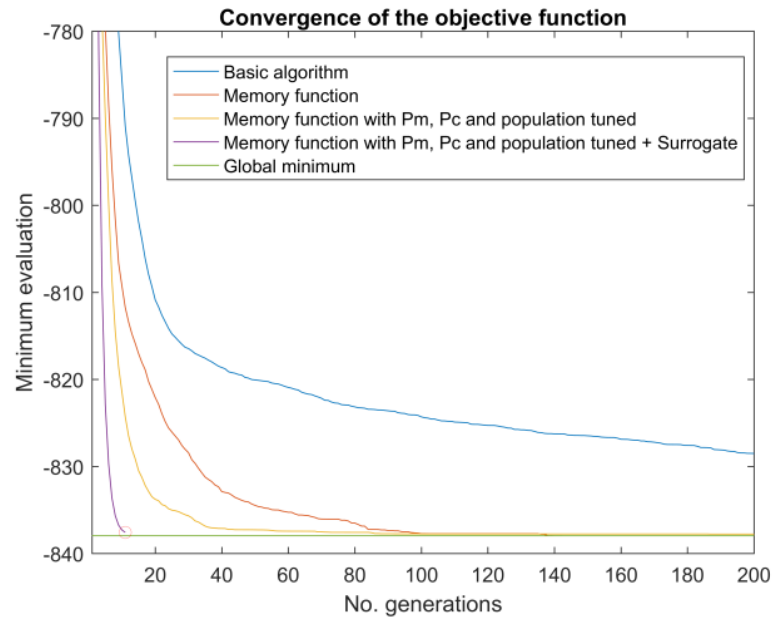

(b)

Figure 11: The Schwefel Function (a) and the average convergence of the genetic algorithm of 1000 different optimisations (b).

\subsection{The genetic algorithm optimisation}

The optimisation of the multi-float configurations considers the genetic algorithm combined with all the improvements discussed in the previous section. The influence of the surrogate model on the genetic algorithm is determined by the use of an elitism coefficient of $20 \%$ of the population.

The population number is fixed as 5 times the number of variables while the total number of generations is fixed to 5 due to computational time reasons. The total number of simulations is the same of the previous Taguchi and Latin hypercube DOE analysis.

Table 5 shows the optimal results of the various configurations. Float spacing generally converges towards lower values but not for the 3 and 4 floats configurations. It is possible that for these 2 configurations the optimisation process has not quite reached the global optimum because of the small number of genetic algorithm generations. Float-reactor separation converge in the lower range as well as the PTO stiffness and damping. Finally the LCOE is decreased from a single float configuration to a 6 float configuration by $21 \%$. Figure 12 shows the increase of the capacity factor with an increase of the number of floats per device. The decrease of the LCOE with an increase the number of floats per device is shown in Figure 13. An interesting results is that the 6 floats configuration shows an increase of the CAPEX compared to the 5 floats configuration but there is also an increase of the capacity factor with the overall result of a lower LCOE. More specifically, the CAPEX of the 6 floats configuration is larger compared to the 5 floats one because its structure cost is higher due to the larger float spacing. Figure 13 shows also that the main CAPEX decrease is until a configuration of 3 floats. In fact, CAPEX costs apart structure and PTO are reduced with the number of devices and the relative decrease of these costs is larger for smaller multi-float configurations. Finally, the 6 floats configuration demonstrated the lowest LCOE, so configurations with a larger number of floats would be interesting to investigate. However, the 
relative decrease of the LCOE is quite slight for the largest number of floats considered and so the benefit will be probably small. Moreover, 2 main limitations stopped the investigation of larger multi-float configurations. The first is related to the computational time required that becomes very high. The second is a practical limitation because the device becomes unmanageably long for multi-float configurations larger than 6 .

Figure 14 shows the sensitivity study of the LCOE for a 6 floats configuration in a similar way of Figure 10. The design parameters are the PTO stiffness and damping while the other 2 parameters are fixed to the optimal case of the genetic algorithm. Similar conclusions of the Latin hypercube DOE sensitivity study can be obtained. However the evaluation points are more concentrated in this case around the optimal value because they are selected through the use of the genetic algorithm. A complete convergence is not expected due to the limited number of evaluations but this method enables refinement of the optimum LCOE found compared to the Taguchi and Latin hypercube DOE. Table 5 shows the NRMSE and the MAX error of the surrogate model and they are calculated considering the predictability on the last generation of the genetic algorithm. The surrogate model is constructed for this calculation on the first 4 generations simulation cases.

To draw these approaches together, a summary of the optimum LCOE obtained from the different methods is shown in Figure 15. The optimum found from the surrogate models described by all the simulation points of the Latin hypercube DOE and the genetic algorithm has been also added in the comparison but they don't obtain a better optimum because the surrogate model is not accurate enough. The optimum found by the genetic algorithm is demonstrated to be the most efficient to find the minimum LCOE from all the methods used. It is also clear that configurations with a number of floats larger than 3 will have only a slight reduction of the minimum LCOE.

Table 5: The design parameters and main results for each multi-float WaveSub configuration derived for the optimum obtained from the GA optimisation. The LCOE and the CAPEX have been normalized to the maximum value obtained.

\begin{tabular}{|l|l|l|l|l|l|l|}
\hline Number of floats & $\mathbf{1}$ & $\mathbf{2}$ & $\mathbf{3}$ & $\mathbf{4}$ & $\mathbf{5}$ & $\mathbf{6}$ \\
\hline Float spacing (m) & - & 46 & 101 & 94 & 39 & 53 \\
\hline Float-reactor Sep (m) & 45 & 44 & 33 & 34 & 36 & 29 \\
\hline kPTO (kN/m) & 58 & 74 & 10 & 14 & 10 & 10 \\
\hline cPTO (kNs/m) & 203 & 370 & 177 & 251 & 337 & 206 \\
\hline RatedP (MW) & 1.02 & 1.72 & 3.19 & 4.10 & 4.10 & 4.91 \\
\hline Energy device (GWh/y) & 3.9 & 6.8 & 12.8 & 16.5 & 16.6 & 20.6 \\
\hline Ndevices & 393 & 232 & 126 & 98 & 98 & 82 \\
\hline CF & 0.44 & 0.45 & 0.46 & 0.46 & 0.46 & 0.48 \\
\hline CAPEX (-) & 1.000 & 0.960 & 0.885 & 0.882 & 0.877 & 0.903 \\
\hline
\end{tabular}




\begin{tabular}{|l|c|c|c|c|c|c|}
\hline CAPEX-Structure (-) & 0.427 & 0.489 & 0.479 & 0.493 & 0.489 & 0.525 \\
\hline CAPEX-PTO (-) & 0.321 & 0.321 & 0.323 & 0.323 & 0.323 & 0.323 \\
\hline CAPEX-Control (-) & 0.052 & 0.031 & 0.017 & 0.013 & 0.013 & 0.011 \\
\hline CAPEX-Grid (-) & 0.051 & 0.031 & 0.017 & 0.013 & 0.013 & 0.012 \\
\hline CAPEX-Mooring (-) & 0.034 & 0.020 & 0.011 & 0.009 & 0.009 & 0.007 \\
\hline CAPEX-Installation (-) & 0.011 & 0.007 & 0.005 & 0.004 & 0.004 & 0.004 \\
\hline CAPEX-Margin (-) & 0.103 & 0.061 & 0.033 & 0.026 & 0.026 & 0.022 \\
\hline OPEX (y $\left.{ }^{-1}\right)$ & 0.008 & 0.006 & 0.004 & 0.004 & 0.004 & 0.004 \\
\hline Decommissioning (-) & 0.005 & 0.003 & 0.002 & 0.001 & 0.001 & 0.001 \\
\hline LCOE (-) & 1.00 & 0.92 & 0.82 & 0.81 & 0.80 & 0.79 \\
\hline NRMSE (-) & 0.15 & 0.03 & 0.04 & 0.10 & 0.02 & 0.11 \\
\hline MAX (-) & 1.00 & 3.04 & 1.71 & 3.52 & 0.98 & 6.68 \\
\hline
\end{tabular}

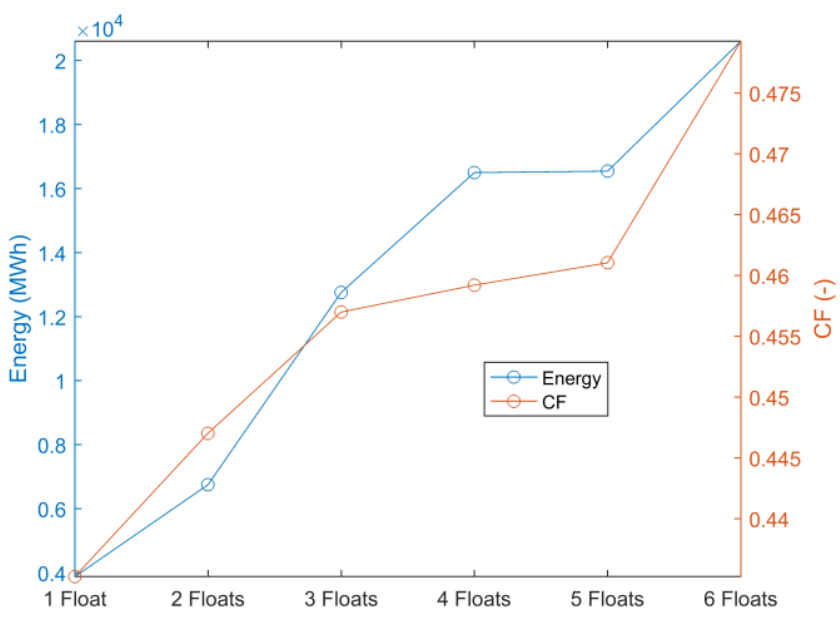

Figure 12: The energy produced and the capacity factor for the optimal cases given by the GA optimisation. 
472

473

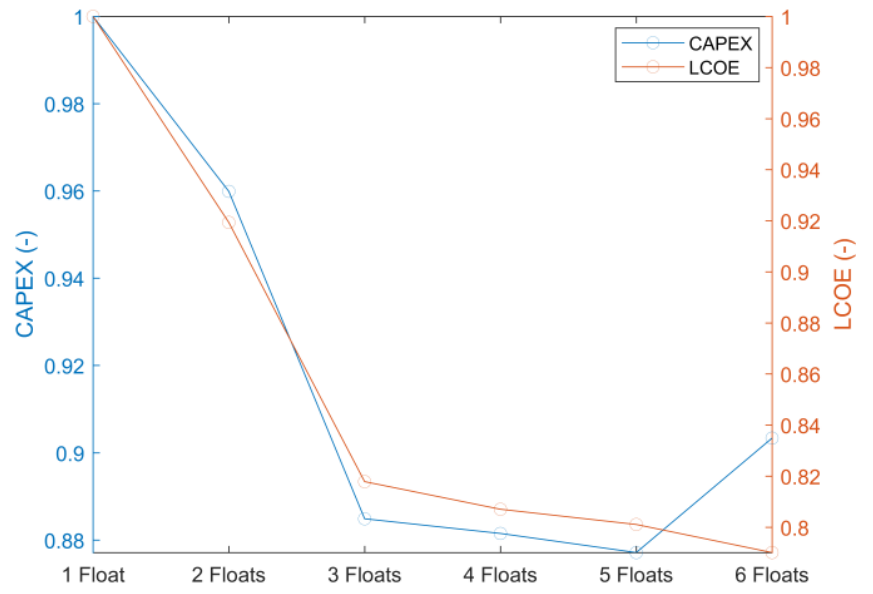

Figure 13: The normalized CAPEX and the LCOE for the optimal cases given by the GA optimisation.

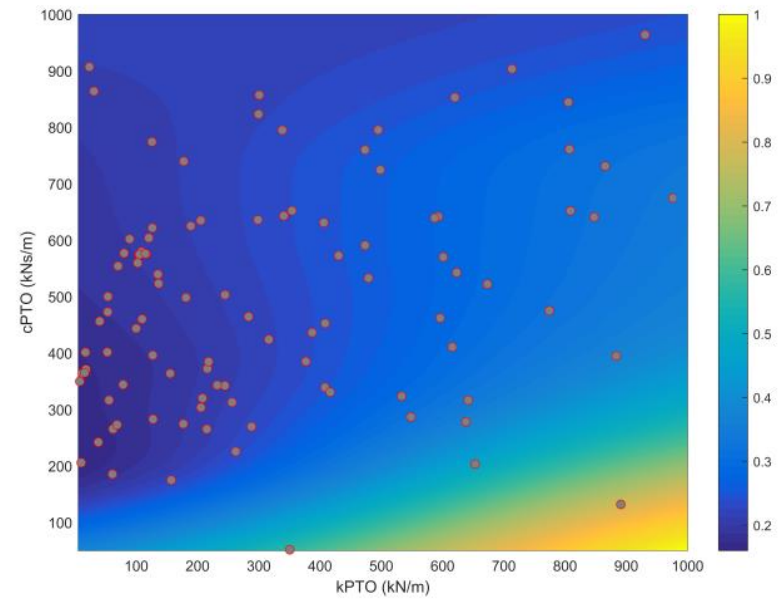

475 Figure 14: The surrogate model for the optimal case of the GA optimisation for a 6 float configuration. PTO stiffness and damping are

476 investigated while the other 2 parameters are fixed to the optimal case. Darker blue shows where the optimal values of the LCOE are expected. 


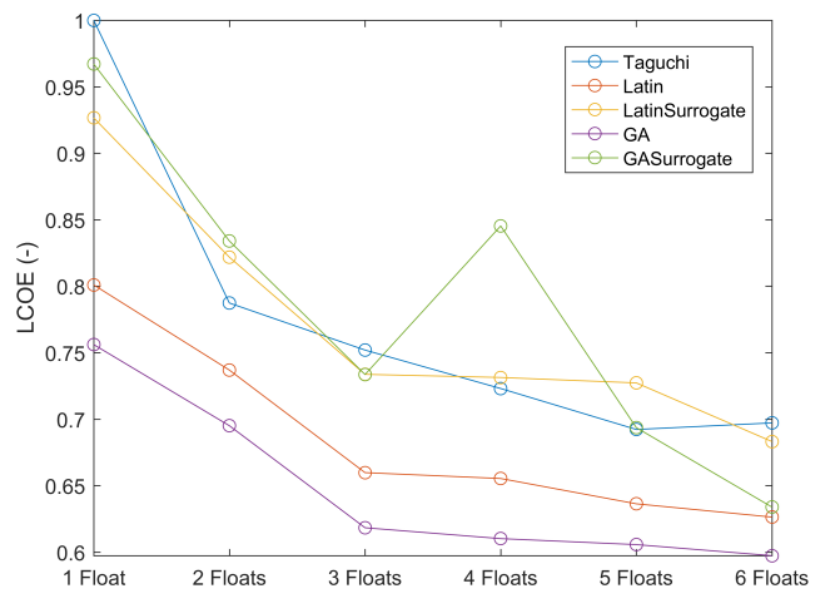

Figure 15: The optimum LCOE summary for all of the methods considered.

\section{Discussion}

The results of the optimisation relies on the accuracy of the estimation of the drag coefficient. This estimation was based on (Faraggiana et al., 2020) but it was limited to regular waves and to a 3 multi-float configuration. A good benchmarking between experimental and numerical data was obtained for drag coefficients in a small data range and so a constant drag coefficient value is reasonably accurate. There is, however, the assumption that the variation of the drag coefficient dependent on the float spacing, the float reactor separation and the float number is reasonably low. This assumption enables to reduce significantly the simulation time because the theory can be simplified using a potential flow theory instead of using CFD. This reduction in the simulation time is also very essential for the application of the optimisation algorithm.

PTO and mooring forces have been simplified with a spring-damper and a spring respectively to reduce the computational time and the complexity involved in the optimisation. However, a more realistic PTO should take in account the designed hydraulic PTO system of the WaveSub device (Hillis et al., 2020). The PTO force of an hydraulic system was obtained using a Coulomb damping model in (Babarit et al., 2012) to approximate the force given by the high and the low pressure accumulators. However, a linearised relationship between the PTO force and the line velocity could be obtained using a control system. The control system will give the required value of PTO force to obtain the optimal power produced from the device. As a result, the linearisation of the PTO will still give a good first approximation of the power produced. Large movements of the floats are expected and the PTO movement is designed to avoid any limitations of end stops in the system. The simulation of the mooring system is another important component of a WEC especially in terms of reliability of the device. However, it is not expected that this will have a large influence on the power produced from the WaveSub device. High tensile forces are designed for the reactor component and this will consequently create a very steady structure. A taut mooring is foreseen for the WaveSub device and it could be reasonably 
linearised in the numerical model as demonstrated in (Faraggiana et al., 2020). More complexity would be involved if MPS had chosen a catenary mooring design.

The sensitivity analysis has been obtained using a Taguchi and a Latin hypercube DOE. These methods are very powerful tools to understand the influence of the design parameters on the LCOE. The Taguchi DOE, however, doesn't consider the interaction between the parameters on the response. The Latin hypercube DOE, instead, can account the interactions between the design variables but it is limited to the efficiency of the surrogate model in the prediction of the unknown response. Other techniques can be also used instead of a Latin hypercube DOE with a similar efficiency in terms of equidistribution of the simulation points, such as the Sobol sequence (Cavazzuti, 2012).

The genetic algorithm has been used for the optimisation of the linear WaveSub configurations mainly because of its known capability and because the information of the derivate is now available. Improvements of this open-source algorithm applied in this research, can be understood more deeply, for example investigating more test functions. However this was not the main purpose of this research and so the time dedicated for this investigation has been limited to a reasonable satisfaction.

The genetic algorithm has been limited to only 5 number of generations and this choice will have certainly some consequences in terms of convergence to the optimal solution. The main aim of the use of this algorithm was not to find the global optimum but to refine the optimal solution of the Latin and Taguchi design of experiments. In-fact these 2 methods already give a good estimate of LCOE between the multi-float configurations.

\section{Conclusions}

This paper is concerned with finding the lowest cost of energy from an array of multi-float wave energy devices. LCOE is the most appropriate metric to consider as it incorporates both power production and cost. A farm of 400MW of linear multi-float WECs has been modelled and optimised using three methods. A sensitivity study was first undertaken using a DOE approach and two techniques were compared, Taguchi and Latin hypercube. Subsequently a genetic algorithm optimisation has been applied to make a refinement of the optimal configuration. The results of these studies were used to create a surrogate model to understand the shape of the optimal LCOE surface.

Sensitivity studies give similar conclusions regarding the optimal range of the design parameters. They agree in the convergence of the PTO stiffness towards lower values. Float spacing generally converges towards lower values while the float-reactor separation converges towards the low-medium range. In addition, the optimal PTO damping is generally in the medium range but it shows less convergence compared to the other ones. The Taguchi DOE shows that the average influence of each design parameter in the LCOE is very similar between the multi-float configurations. The Latin hypercube DOE is used mainly to build the Kriging surrogate model and to analyse the influence of design parameters combined together. Finally the genetic algorithm optimisation 
show the optimal design parameters for each multi-float configuration. There is clearly a reduction in optimal LCOE with an increase of the float number per device which is driven by a reduction of the CAPEX and an increase of the capacity factor. The reduction of the CAPEX is mainly due to the reduction of the number of devices for larger multi-float configurations with the same total rated power of the wave farm. In fact, the control, grid, mooring, installation and margin costs are reduced if the number of devices is decreased. On the other side the increase of the capacity factor with larger multi-float configurations has determined a larger total amount of energy from the WEC farm with a consequent lower LCOE. However, the reduction of the LCOE is small for multi-float configurations larger than 3. The reduction of the LCOE from the single float configuration to the 6 float configuration is around $21 \%$.

Further work will include an extension of the optimisation for different arrangements of the floats in addition to the linear shape considered here. Additionally, a calculation of the energy production of the wave farm that takes in account the hydrodynamic interactions between the WECs could be also considered. Finally, a non-uniform spacing between the floats will be investigated because it could increase further the energy produced by each WEC.

\section{Acknowledgments}

This research is supported by the Knowledge Economy Skills Scholarships (KESS 2). It is a pan-Wales higher level skills initiative led by Bangor University on behalf of the HE sector in Wales. It is part funded by the Welsh Government's European Social Fund (ESF) convergence programme for West Wales and the Valleys. The work was also supported by the SURFTEC SuperGen grand challenge project, funded under EPSRC grant EP/N02057X/1.

\section{References}

Aqua-RET (2019) Wave. Available at: http://www.aquaret.com/images/stories/aquaret/pdf/chapter4.pdf.

Babarit, A., Hals, J., Muliawan, M. J., Kurniawan, A., Moan, T. and Krokstad, J. (2012) 'Numerical benchmarking study of a selection of wave energy converters', Renewable energy. Elsevier, 41, pp. 44-63.

Babarit, A. (2017) Nemoh. Available at: https://heea.ec-nantes.fr/logiciels-et-brevets/nemoh-presentation-192863.kjsp. Blasco, X. (2019) Genetic algorithm. Available at: https://uk.mathworks.com/matlabcentral/fileexchange/39021-basicgeneticalgorithm.

Borgarino, B., Babarit, A. and Ferrant, P. (2012) 'Impact of wave interactions effects on energy absorption in large arrays of wave energy converters', Ocean Engineering. Elsevier, 41, pp. 79-88.

Budal, K. (1977) 'Theory for absorption of wave power by a system of interacting bodies', Journal of Ship Research. The Society of Naval Architects and Marine Engineers, 21(04), pp. 248-254. 
558

Cavazzuti, M. (2012) Optimization methods: from theory to design scientific and technological aspects in mechanics. Springer Science \& Business Media.

Chakrabarti, S. (2005) 'Hybrid numerical method for wave-multibody interaction', WIT Transactions on State-of-the-art in Science and Engineering. WIT Press, 18.

Chapman, J., LeDreff, J., Foster, G., Stockman, G. and Brask, A. (2017) 'Improving Energy Capture by Varying the Geometry of a Novel Wave Energy Converter', in Proceedings of the 12th European Wave and Tidal Energy Conference, Cork, Ireland.

Child, B. F. M. (2011) On the configuration of arrays of floating wave energy converters. $\mathrm{PhD}$ thesis. The University of Edinburgh.

Child, B. F. M. and Venugopal, V. (2009) 'Modification of power characteristics in an array of floating wave energy devices', in Proceedings of the 8th European Wave and Tidal Energy Conference, Uppsala, Sweden, pp. 309-318.

Codeaster (2019) Salome-meca. Available at: http://www.code-aster.org/spip.php?article303.

Couckuyt, I., Dhaene, T. and Demeester, P. (2014) ‘ooDACE toolbox: a flexible object-oriented Kriging implementation', The Journal of Machine Learning Research. JMLR. org, 15(1), pp. 3183-3186.

Cruz, J. (2007) Ocean wave energy: current status and future prespectives. Springer Science \& Business Media.

Delhommeau, G. (1993) 'Seakeeping codes aquadyn and aquaplus', 19th WEGEMT School Numerical Simulation of Hydrodynamics: Ships and Offshore Structures.

Falcão, A. F. O. (2014) ‘Modelling of wave energy conversion’, Instituto Superior Técnico, Universidade Técnica de Lisboa. Faraggiana, E., Masters, I., Chapman, J., Foster, G. and Stockman, G. (2019) ‘Multi-directional waves and time domain perturbed field visualization of the WaveSub device', in Proceedings of the 13th European Wave and Tidal Energy Conference, Napoli, Italy.

Faraggiana, E., Whitlam, C., Chapman, J., Hillis, A., Roesner, J., Hann, M., Greaves, D., Yu, Y.-H., Ruehl, K. and Masters, I. (2020) 'Computational modelling and experimental tank testing of the multi float WaveSub under regular wave forcing', Renewable Energy. Elsevier.

Faraggiana, E., Masters, I. and Chapman, J. (2018) 'Design of an optimization scheme for the wavesub array', in Proceedings of 3rd International Conference on Renewable Energies Offshore, Lisbon, Portugal.

Giassi, M., Göteman, M., Thomas, S., Engström, J., Eriksson, M. and Isberg, J. (2017) 'Multi-parameter optimization of hybrid arrays of point absorber Wave Energy Converters', in 12th European Wave and Tidal Energy Conference (EWTEC), Cork, Ireland, August 27-31, 2017.

González-Gorbeña, E., Qassim, R. Y. and Rosman, P. C. C. (2018) 'Multi-dimensional optimisation of tidal energy converters array layouts considering geometric, economic and environmental constraints', Renewable energy. Elsevier, 116, pp. 647-658. 
588 Göteman, M., Engström, J., Eriksson, M. and Isberg, J. (2015) 'Fast modeling of large wave energy farms using interaction 589 distance cut-off', Energies. Multidisciplinary Digital Publishing Institute, 8(12), pp. 13741-13757.

590 Göteman, M. (2017) 'Wave energy parks with point-absorbers of different dimensions', Journal of Fluids and Structures. $591 \quad$ Elsevier, 74, pp. 142-157.

592 Hillis, A. J., Whitlam, C., Brask, A., Chapman, J. and Plummer, A. R. (2020) 'Active control for multi-degree-of-freedom wave 593 energy converters with load limiting', Renewable Energy. Elsevier.

594 Holthuijsen, L. H. (2010) Waves in oceanic and coastal waters. Cambridge university press.

595 Ireland Ocean Energy Expertise (2019) Atlantic marine energy test site (full scale). Available at:

596 http://www.oceanenergyireland.ie/TestFacility/AMETS.

597 Leung, Y.-W. and Wang, Y. (2001) 'An orthogonal genetic algorithm with quantization for global numerical optimization', 598 IEEE Transactions on Evolutionary computation. IEEE, 5(1), pp. 41-53.

599 Mahmoodabadi, M. J. and Nemati, A. R. (2016) 'A novel adaptive genetic algorithm for global optimization of mathematical test 600 functions and real-world problems', Engineering Science and Technology, an International Journal. Elsevier, 19(4), pp. 20026012021.

602 MATLAB (2019a) Orthogonal array. Available at: https://uk.mathworks.com/matlabcentral/fileexchange/47218-orthogonal603 array.

604 MATLAB (2019b) Parfor function. Available at:

605 https://www.mathworks.com/help/parallelcomputing/parfor.html;jsessionidÆ663e0ead6e3c90409fe51a99883a.

606 Mavrakos, S. A. (1991) 'Hydrodynamic coefficients for groups of interacting vertical axisymmetric bodies', Ocean Engineering. 607 Elsevier, 18(5), pp. 485-515.

608 Mavrakos, S. A. and Koumoutsakos, P. (1987) 'Hydrodynamic interaction among vertical axisymmetric bodies restrained in 609 waves', Applied Ocean Research. Elsevier, 9(3), pp. 128-140.

610 Mavrakos, S. A. and McIver, P. (1997) 'Comparison of methods for computing hydrodynamic characteristics of arrays of wave 611 power devices', Applied Ocean Research. Elsevier, 19(5-6), pp. 283-291.

612 McGuinness, J. P. L. and Thomas, G. (2016) 'Hydrodynamic optimisation of small arrays of heaving point absorbers', Journal of 613 Ocean Engineering and Marine Energy. Springer, 2(4), pp. 439-457.

614 McNatt, J. C. (2019) mwave. Available at: https: //github.com/cmcnatt/mwave.

615 McNatt, J. C., Venugopal, V. and Forehand, D. (2013) 'The cylindrical wave field of wave energy converters', International 616 Journal of Marine Energy. Elsevier, 3, pp. e26--e39.

617 Neill, S. P. and Hashemi, M. R. (2018) Fundamentals of Ocean Renewable Energy: Generating Electricity from the Sea. 
618 Academic Press.

619 Noad, I. F. and Porter, R. (2015) 'Optimisation of arrays of flap-type oscillating wave surge converters', Applied Ocean

620 Research. Elsevier, 50, pp. 237-253.

621 Roy, R. K. (2010) A primer on the Taguchi method. Society of Manufacturing Engineers.

622 Sarkar, D., Renzi, E. and Dias, F. (2014) 'Wave farm modelling of oscillating wave surge converters', Proceedings of the Royal

623 Society A: Mathematical, Physical and Engineering Sciences. The Royal Society Publishing, 470(2167), p. 20140118.

624 Sharp, C., DuPont, B., Bosma, B., Lomonaco, P. and Batten, B. (2017) 'Array Optimization of Fixed Oscillating Water Columns 625 for Active Device Control', in Proceedings of the 12th European Wave and Tidal Energy Conference.

626 Stratigaki, V. (2014) Experimental study and numerical modelling of intra-array interactions and extra-array effects of wave 627 energy converter arrays. PhD thesis. Ghent University.

628 Yarpiz (2019) Particle swarm optimisation. Available at: https://yarpiz.com/50/ypea102-particle-swarm-optimization (Accessed: 6293 December 2019).

630 Yu, Y.-H., Ruehl, K., Van Rij, J. and Tom, N. (2020) WEC-Sim. Available at: https://wec-sim.github.io/WEC-Sim/ (Accessed: 63130 March 2020).

632 Zhang, W., Liu, H., Zhang, X., Zhang, L. and Ashraf, M. A. (2016) 'Optimal configurations of wave energy converter arrays 633 with a floating body’, Polish Maritime Research. De Gruyter Open, 23(s1), pp. 71-77. 